This item was submitted to Loughborough's Research Repository by the author.

Items in Figshare are protected by copyright, with all rights reserved, unless otherwise indicated.

\title{
Photomaps: A visual taxonomy
}

PLEASE CITE THE PUBLISHED VERSION

https://doi.org/10.1177/1470357217746028

PUBLISHER

SAGE Publications

VERSION

AM (Accepted Manuscript)

PUBLISHER STATEMENT

This work is made available according to the conditions of the Creative Commons Attribution-NonCommercialNoDerivatives 4.0 International (CC BY-NC-ND 4.0) licence. Full details of this licence are available at: https://creativecommons.org/licenses/by-nc-nd/4.0/

\section{LICENCE}

CC BY-NC-ND 4.0

\section{REPOSITORY RECORD}

Tovey, Robert. 2019. "Photomaps: A Visual Taxonomy". figshare. https://hdl.handle.net/2134/36653. 


\section{KEY WORDS}

photography • mapping • photomap • information design - representation • digital composite

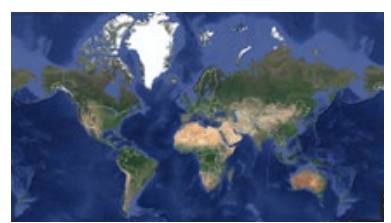

Fig.1

Google Maps satellite view features hundreds of photographs composited together following Mercator's projection.

\section{PHOTOMAPS:}

\section{A VISUAL TAXONOMY}

Multiple viewpoint photo composites, or photomaps, are commonplace, but critically and popularly overlooked. They bring together principles from information graphics and geographic visualisation and combine them with the image-making technologies of photography. Well known examples include the ubiquitous panorama and aerial composites such as Google Map's satellite view (fig.1). Forerunners are as old as photography itself including examples from Henry Fox-Talbot and Eadweard Muybridge.

In this visual essay the formal possibilities of photomaps are explored through practical enquiry. Alternate visual schemas are used to arrange photographic information with the purpose of setting out some of the parameters and possibilities of this mode of representation. What follows is a visual taxonomy of photomaps covering cartographic, scanned, diagrammatic, peripheral and topological approaches. This is not an exhaustive list by any means but does set out the territory for what has been an under-theorised visual approach.

Texts on the subject are limited, with the majority focusing on technological innovations in optics and compositing, geographic information systems (GIS) and, less often, Google Earth. The only major texts to explicitly address photo-composite representations include Nicholas Felton's recent PhotoViz: Visualizing information through photography (2016) and Dawn Ades' Photomontage (1976), both of which have a very different emphasis to this study.

Rather than frame the photomap as an isolated visual mode, it is conceptualised here as being part of an expanded field of visual media, which merges into photography, film, digital imagery and information graphics. George Baker in 'Photography's Expanded Field' argues that the blurring of boundaries between diverse visual forms has meant 'photography has been thoroughly transformed today' but that 'something like a photographic effect still remains' (2005: 123). This is echoed in statements from Costello (2007), Fried (2008) and Green and Lowry (2005). This 'photographic effect' is present, and indeed fundamental, to photomaps. Baker sets out an expansive structure, arguing: 'Perhaps, indeed, photography's expanded field... might even have to be imagined as a group of expanded fields, multiple sets of oppositions and conjugations, rather than any singular operation' (ibid: 124 , his emphasis). It is within this framework that the study is situated, with the "photomap" at that liminal point between photography, information graphics and mapping, and it is the parameters of this expanded field that are explored here. 


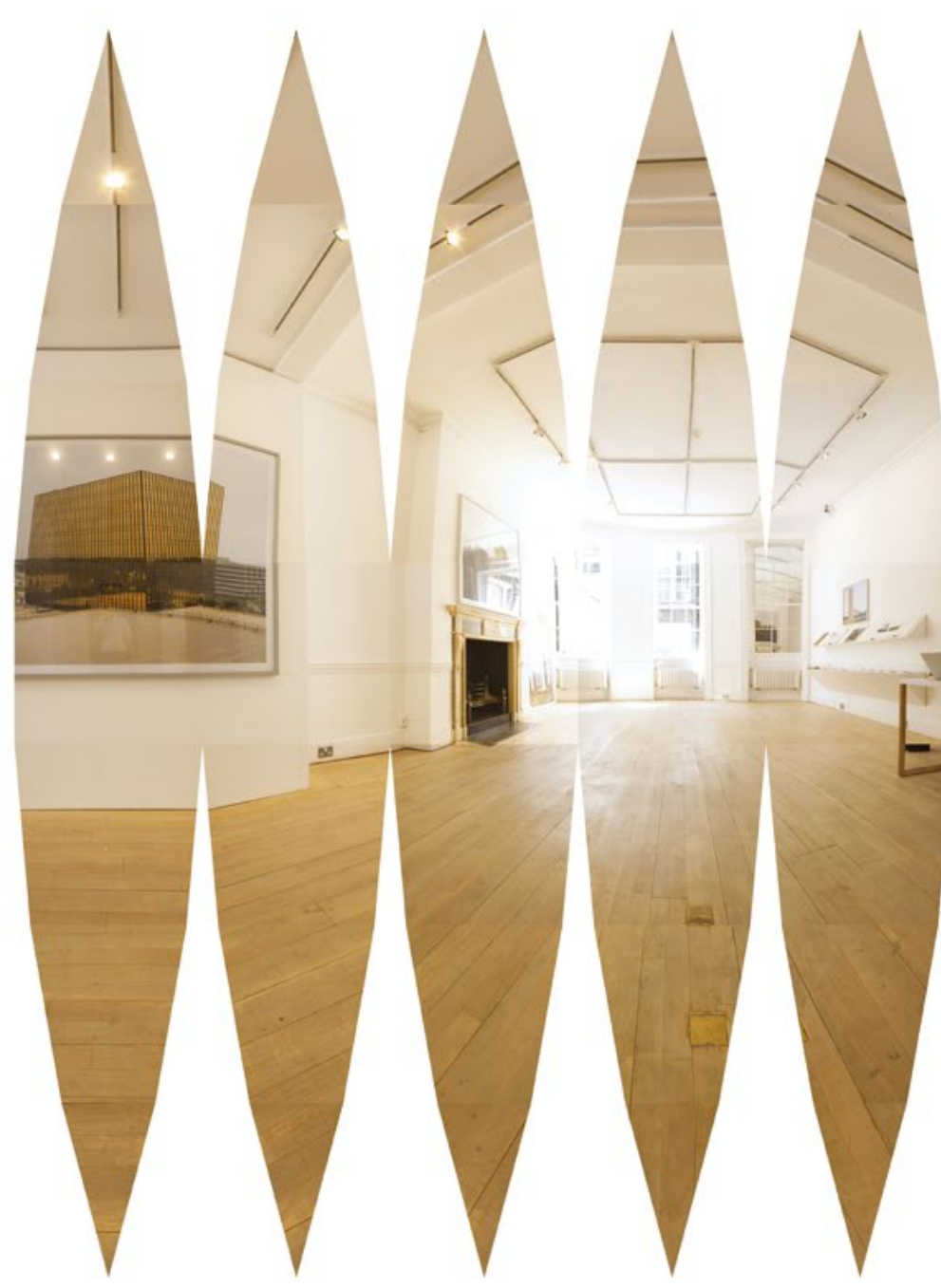

\section{Cartographic Projection}

From any static point looking out in all directions we have, in effect, a sphere-of-vision. This sphere, much like a globe, can be mapped onto a flat plane using a projection. In the imagery shown here various cartographic projections are used to 'map' a sphere-of-vision, taken from the centre point of the Architectural Association gallery.

Considered this way the underlying schem in a panoramic photograph (top right) is similar to a Mercator Projection, translating a sphere into a broad rectangle, and a wide-angle photograph (opposite) uses a similar schema to an Azimuthal Projection, where a hemisphere is translated into a circle. In the imagery above a Graticular Projection is used where the sphere is sliced into twelve double pointed ovals. Unlike wide-angle photography this projection minimises distortion whilst preserving angles between points, but with the effect of visual interrupts.
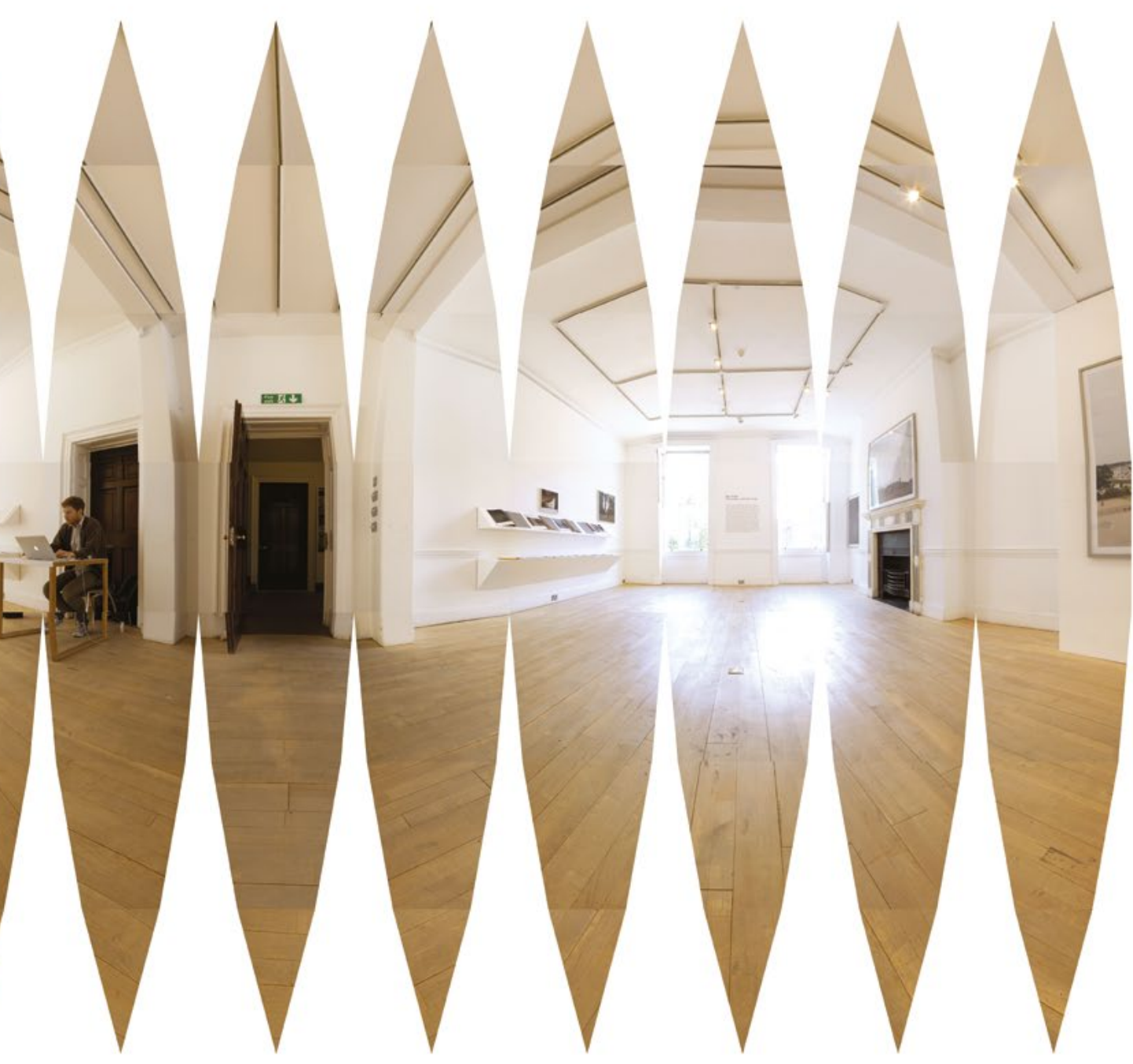

Mercator Projection The image above shares many qualities with Mercator's famous projection of the Earth.
In this the globe is In this the globe is cydinder creating ureelled distortion at the poles where a singular poin is stretched to become a continuous line. The Cylindrical Graticular Projection (left) is similar, with a globe unravelled, but the pointed tips of the graticules mean the top of
the sphere is depicted as a singular point with very little distortion.

Related works David Hockney, The Grand 1982 (1982) Gordon Matta-Clark Gordon Matta-Clark, Sohei Nishino, Diorama (203-2015)

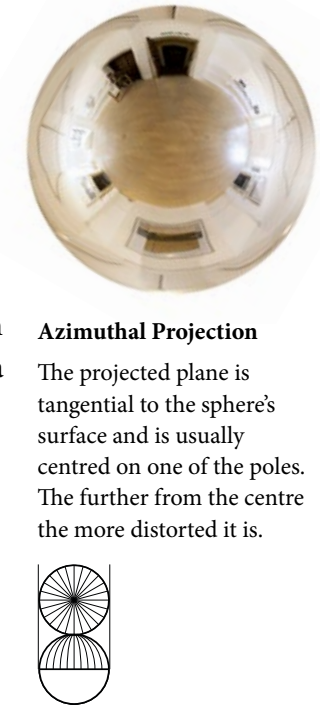

Graticular Photomap of the Architectural Association Gallery (2012), R. Tovey (commumm
Graticular Projections The imagery can be arranged to form various including azimuthal (left), conical (both middle) and cylindrical (right). 


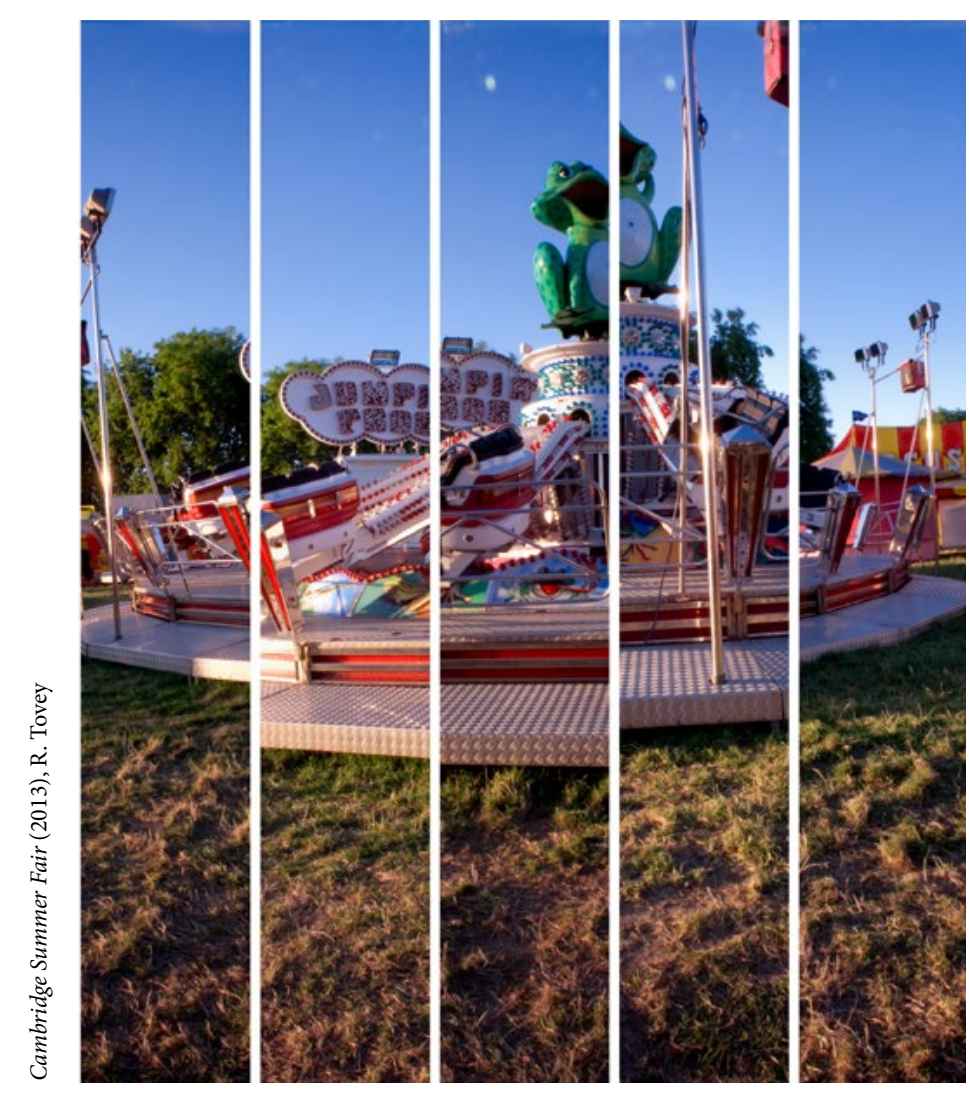

Alignment

Each of the photographs

making up the image have

been cropped to a central

band, a quarter of the

negative's total width.

This crop results in images

is a set distance away from

the camera (at around

10 metres), where all

other distances result in

misalignments. These tak

the form of repetitions for

objects further away and

fragmentary shearing fo

closer objects.

\section{Scanning: Lateral Movement}

An alternate schema is to have the camera itself moving through space, as in, for example, scanning methodologies, where the camera shifts across one plane at a set distance from the subject.

In Cambridge Summer Fair (above), a camera moves in a straight line, perpendicular to the direction it faces. Photographs are taken at 2 metre intervals, with 30-second exposures. The camera travels the length of one of the fairground strips - a wide pathway created by the positioning of fairground attractions. In the process of creating Cambridge Summer Fair the camera moves 144 metres laterally in 73 separate photographs. Across the exposures 31.5 minutes were documented, with short gaps between photographs. The real-world time between the first photograph and the last was 90 minutes, although only a third of that time was recorded in camera.

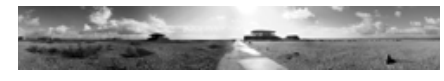

\section{Panorama}

Whilst a panorama has a similar format to a lateral scan, the implied space is
fundamentally different.

Related works

Ed Ruscha, Every Building on the Sunset Strip (1966) Josef Schulz Centro Commercial (1999-2000) Christoph Keller

(various works, 1998-2005)
The Elms, Houghton (2014), R. Tovey

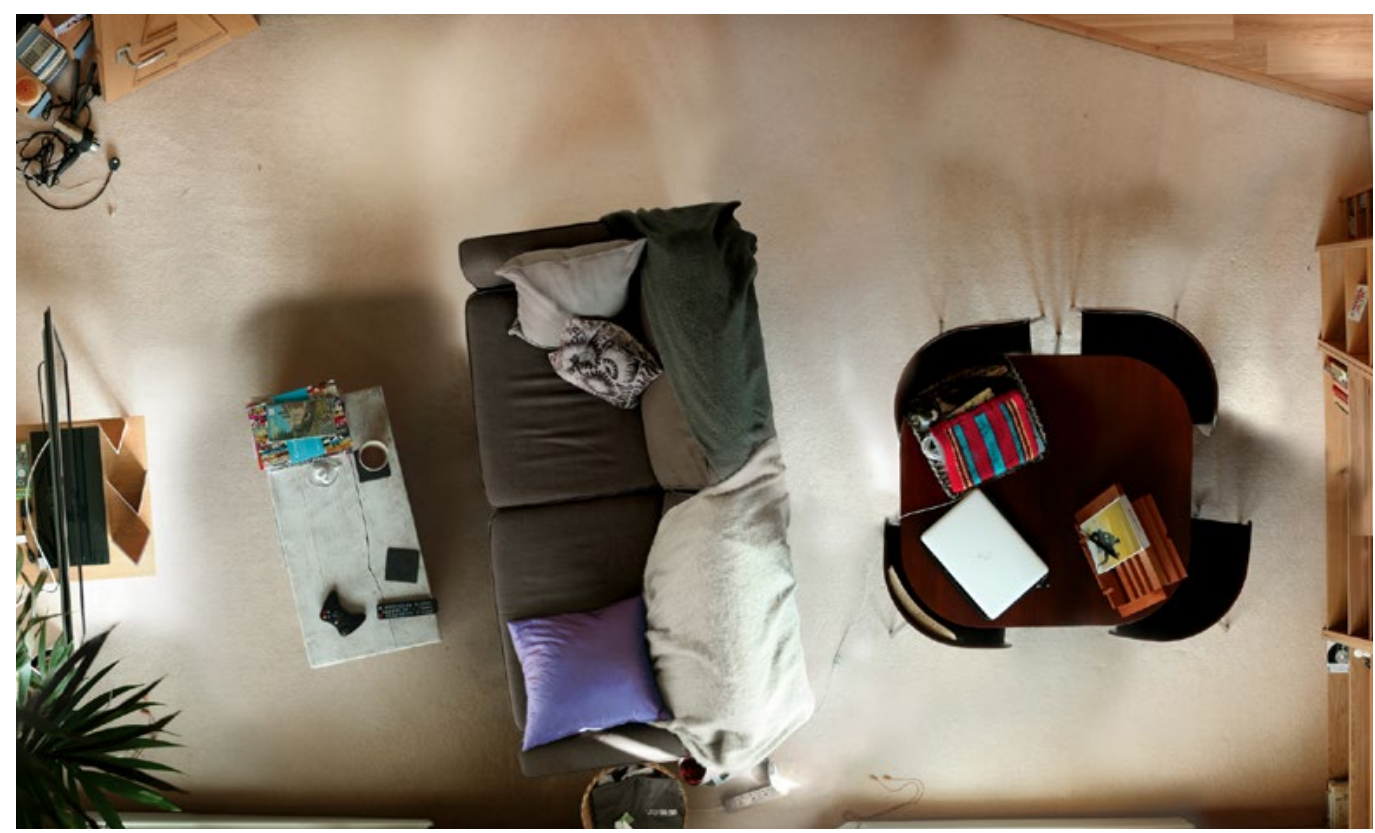

\section{Scanning: Planometric}

In the image above scanning methods are used to move across the surface of the subject, much like a flatbed scanner or photocopier. 54 separate photographs are digitally composited together following a grid schema. The distance between camera and subject is relatively static, meaning the majority of individual photographs align. This falters however, in the top left and bottom right corners where the height of the door and shelves depicted results in a fractured representation that shears into the component photographs. For the majority of the image however, the method of image construction creates the impression that perspective is flattened, with echoes of aerial photography or images captured from distance with a telescopic lens where there is a similar apparent compression of perspective.
Related works

Andreas Gefeller, Aupervisions series Aneta Grzeszykowska an 

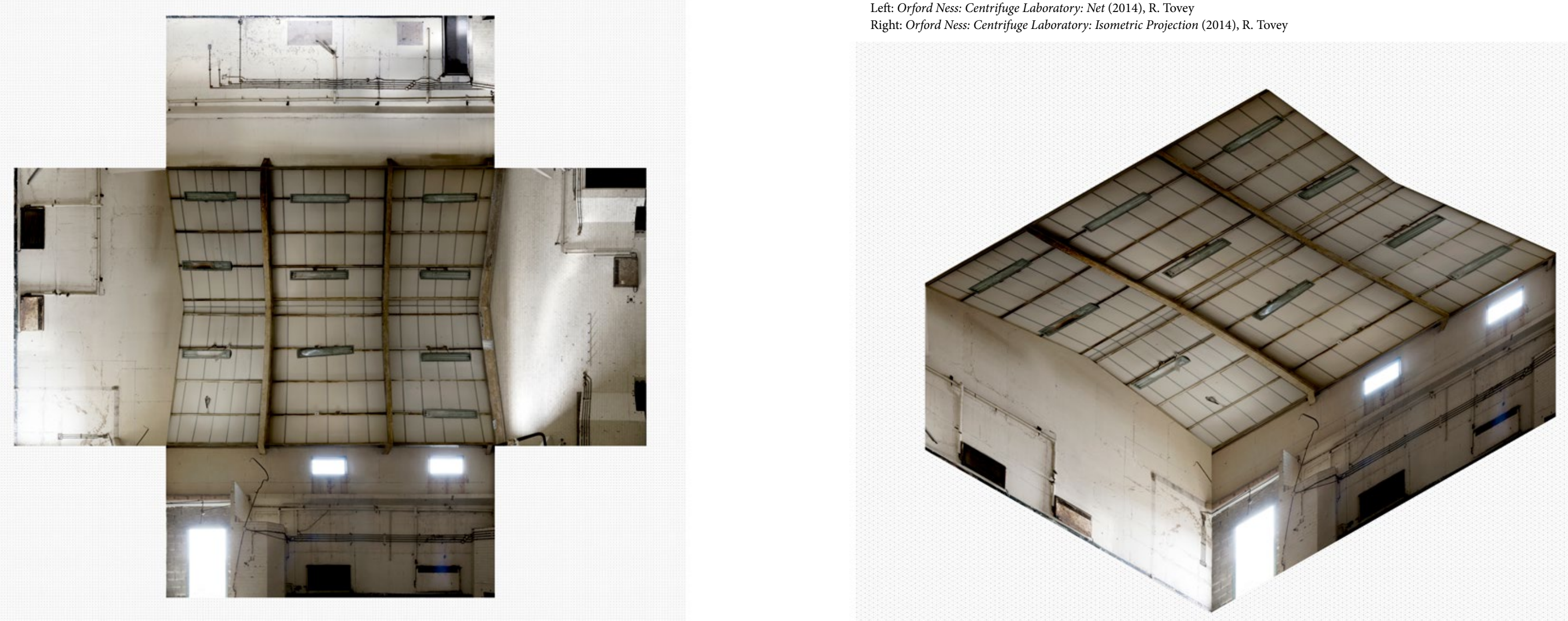

\section{Diagrammatic Approaches}

In the images above the same photographic information is used. The interior of a former military laboratory at Orford Ness was "scanned" with multiple photographs taken at points in a straight line parallel to each wall at three different heights, and following a grid schema to map out the ceiling. Effectively five photomaps were produced, one for each wall and one for the ceiling, each with the flattened perspective seen in scanning approaches. This data was then arranged to produce alternate diagrammatic representations; the first as a geometric net (above) and the second following an isometric projection (right). In the second of these, Orford Ness: Centrifuge Laboratory: Isometric Projection, imagery was then digitally manipulated to follow a 30 degree isometric grid.
Related works

Andreas Gefeller, Supervisions serics
$(2002-2016)$

Aneta Grzeszykowska and Smaga, Plan (2004)
Necker Cube

Most photographs are understood within a where perspective and a knowledge of spatial conventions compels audiences to situate the photographic act in a specific position opposite the subject (the point of view). By producing an isometric photographic image, where the subject
is depicted without perspective, the image

becomes bject-centred. As a result, it is disorienting, almost an optical illusion, like a Necker Cube. 

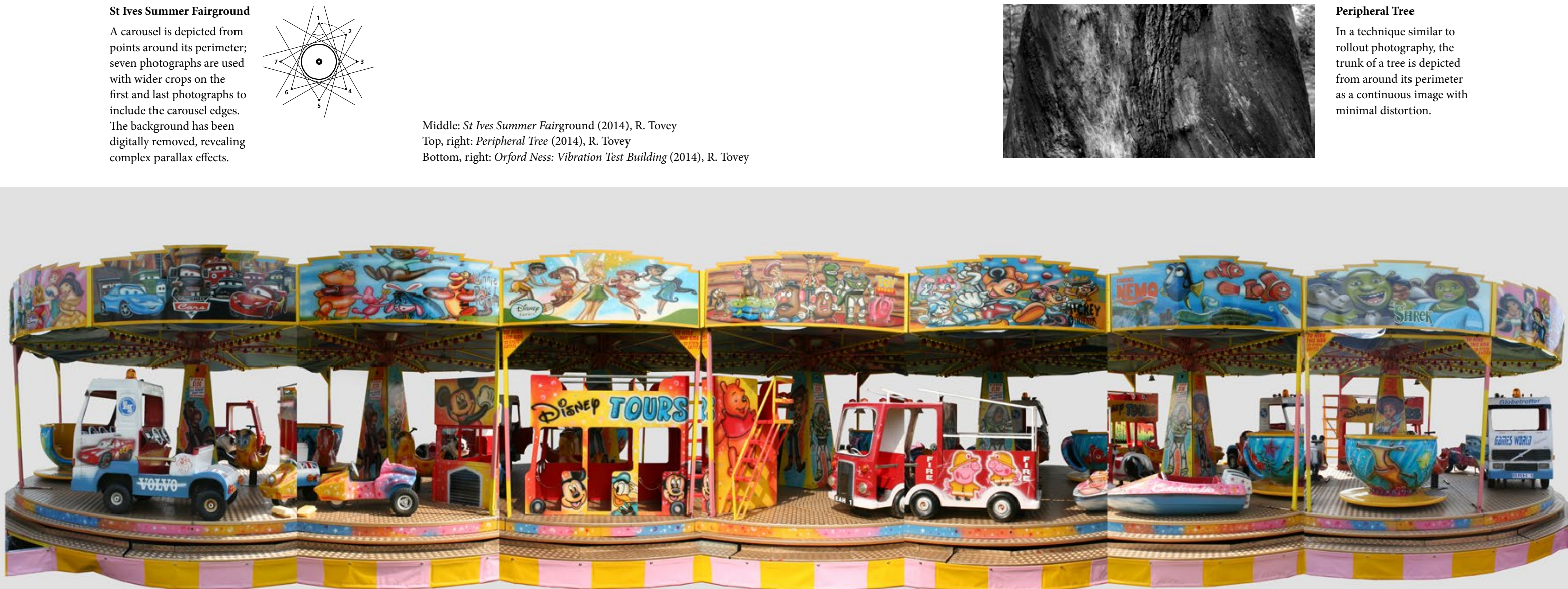

\section{Peripheral Photography}

The technique of photographing a subject from positions around its perimeter has similarities to both diagrammatic approaches and scanning methods. Perspective is distorted, with the point-of-view circling around on itself. Variations in the distance between camera and subject create misalignments between constituent imagery and parallax is a constant factor. The technique is used in a range of niche applications including the archival recording of artifacts in a process known as rollout photography. In this context slit-scan photography is used where the aperture is a thin vertical band which is moved horizontally across the negative as the subject rotates. Rollout photography usually requires the subject to be circular, smal and opaque to avoid distortion and repetition. A broader range of approaches are shown here.

\section{Related works}

Andrew Davidhazy (various works, 1998-2010) Michael D Coe and Ustin Kerr, Lords of the Underworld. Masterpieces of Classic

Tim Macmillan, Time Slice $(1980)$

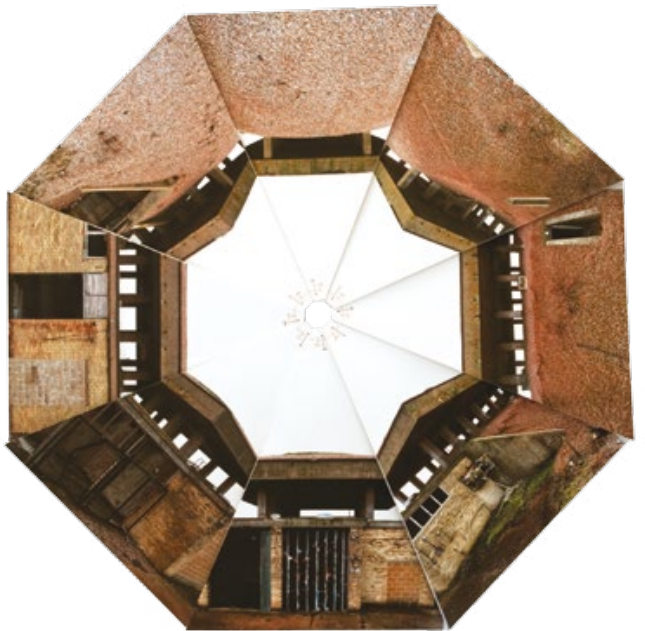

Orford Ness:

ibration Test Building Photographs were taken around the perimeter of the subject. Rather than project the imagery in a line like a panorama the photographic units are oriented relationally. This creates a projection where opposite sides of the with the horizon line rotating round from imas to image. 


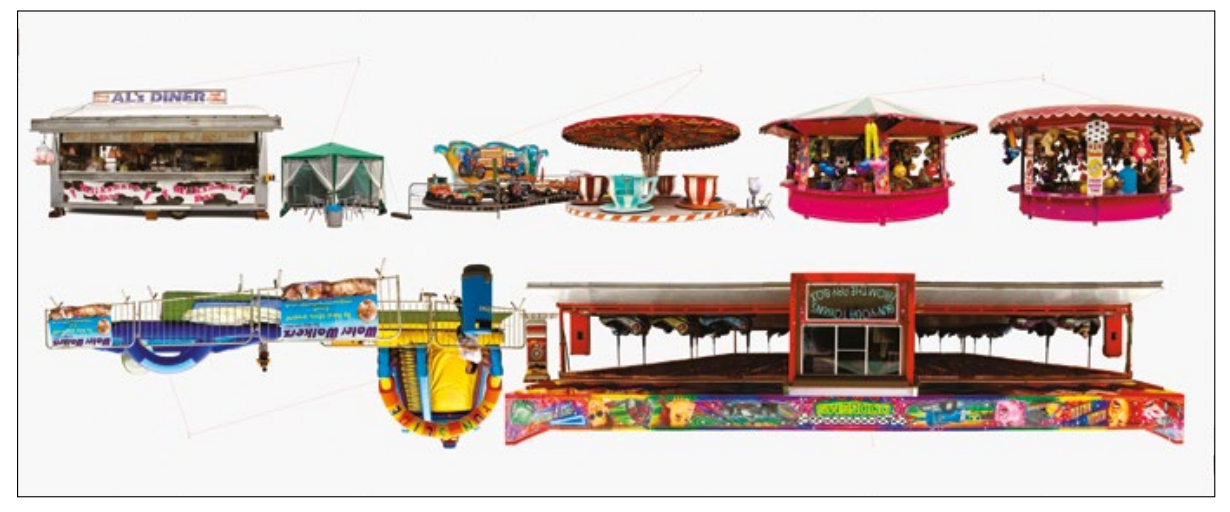

\section{Topological Rationales}

In this final grouping environments are not described as spatial volumes but in terms of the relationships between points. For example, in Alexandre Park Summer Fair (directly above), each of the fairground attractions along one "strip" is depicted as a discrete point, and is arranged next to and opposite the others. Each of the individual attractions has its own local linear perspective. All other visual information is removed isolating the attractions and facilitating their transformation into a network of points. Similarly, in the artwork opposite (crop, right) a journey is depicted from only those moments where the subject is walking. As a result, the movement of vehicles is removed, so that two train platforms become connected by a single train carriage, revealing the experiential reality of a transport network.
Related works

Gordon Matta-Clark Number 26+27'Splitting'

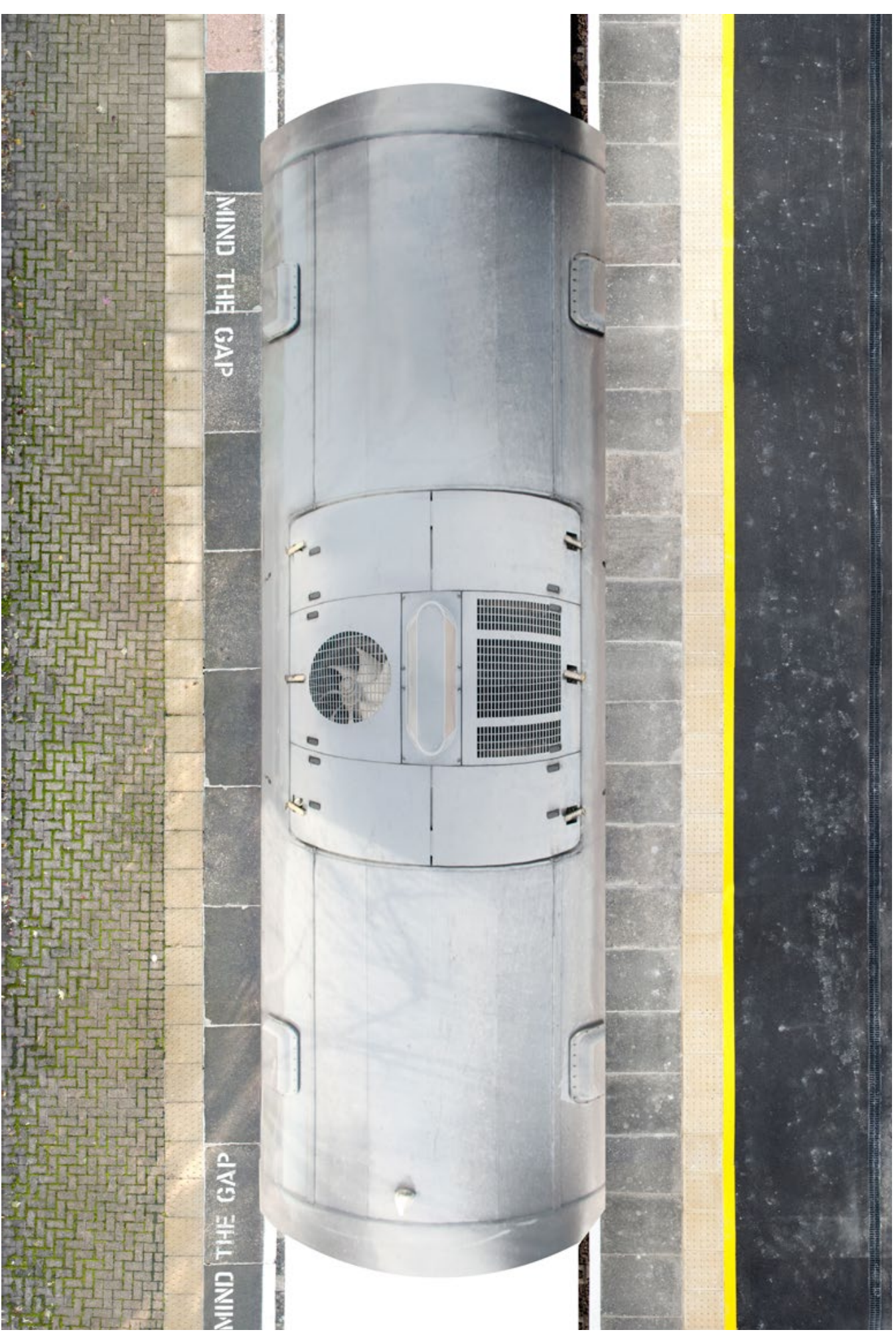




\section{BIOGRAPHICAL NOTE}

DR ROB TOVEY is a Senior Lecturer in Graphic Design at Coventry University. $\mathrm{He}$ is a lead member of the Visual Arts Research Group and a Senior Fellow of the Higher Education Academy. His research investigates photo-composites in the context of information design, mapping and the digital image. He is currently working on funded projects across health, communication design and photography alongside writing various publications. His practice has won and been short-listed for awards including The Searle Prize, Fresh Awards, D\&AD, Digital Innovation Awards and the George Jackson Prize.

Address: Coventry University Priory Street, Coventry, UK, CV1 5FB

Email: rob.tovey@coventry.ac.uk

\section{ACKNOWLEDGEMENTS}

This body of practice would not have been possible without the kind support of the Architectural Association, the National Trust, and various fairground owners.

\section{REFERENCES}

Ades, D. (1976) Photomontage. London: Thames \& Hudson.

Baker, G. (2005) Photography's Expanded Field. October 114 (Fall): 120-40. Costello, D. (2007) After Medium Specificity Chez Fried: Jeff Wall as a Painter; Gerhard Richter as a Photographer. In Photography Theory, ed. J. Elkins, 75-89. New York: Routledge.

Felton, N., S. Ehmann and R. Klanten (2016) PhotoViz: Visualizing Information Through Photography. Berlin: Gestalten.

Fried, M. (2008) Why Photography Matters As Art As Never Before. New Haven: Yale University Press.

Green, D., and J. Lowry, eds. (2005) Stillness and Time: Photography and the Moving Image. Brighton: Photoworks. 